\title{
Ocorrência de sífilis congênita e exposição ao HIV em neonatos atendidos em um hospital materno-infantil
}

\author{
Occurrence of congenital syphilis and exposure to HIV in newborns cared for in a \\ maternal-infant hospital,
}

\begin{abstract}
Aparición de sífilis congénita y exposición al VIH en neonatos atendidos en un hospital materno-infantil
\end{abstract}

\begin{abstract}
Fabiana da Costa Aleixo ${ }^{1}$, Gisele Campos de Oliveira ${ }^{1}$, Maria de Nazaré Santos de Oliveira ${ }^{1}$, Marcos José Risuenho Brito Silva ${ }^{1 *}$, Elisângela Silva Gomes ${ }^{1}$, Jaqueline Pinheiro Morais ${ }^{1}$, Eliseth Costa Oliveira de Matos ${ }^{1}$.
\end{abstract}

\section{RESUMO}

Objetivo: Investigar a prevalência de neonatos com infecção por sífilis e exposição ao HIV atendidos em um hospital público de Belém do Pará. Métodos: Estudo transversal com abordagem quantitativa, descritiva e retrospectiva utilizando dados secundários dos prontuários de pacientes. O número total de neonatos internados no hospital no período em questão foram 8.075 pacientes. Resultados: Neste estudo foram incluídos 312 neonatos com diagnóstico de Sífilis congênita no período do estudo; do total de incluídos, 7,1\% $(n=22)$ foram expostos ao HIV caracterizando a coinfecção. Quanto ao sexo, 53,8\% $(n=167)$ eram do sexo masculino e $75,0 \%(n=234)$ estavam na faixa etária de 0 a 10 dias; considerando a classificação dos casos, 76,0\% ( $\mathrm{n}=237$ ) foram considerados Sífilis Congênita Precoce não Especificada. Conclusão: Com estes dados, conclui-se que a sífilis congênita ainda persiste como problema de saúde pública, associada à maior vulnerabilidade social e falhas na assistência pré-natal.

Palavras-chave: Sífilis Congênita, Infecções por HIV, Recém-Nascido.

\section{ABSTRACT}

Objective: To investigate the prevalence of newborns with syphilis infection and exposure to HIV cared in a public hospital in Belém do Pará. Methods: This is a cross-sectional, descriptive and retrospective study using secondary data from patient records. The total of newborns admitted at the hospital in the surveyed period was 8,075 patients. Results: This study included 312 newborns diagnosed with Congenital syphilis in the research period; of which, $7,1 \%(\mathrm{n}=22)$ were exposed to HIV, thus characterizing the co-infection. Regarding gender, 53.8\% $(n=167)$ were male and $75.0 \%(n=234)$ were in the age range from 0 to 10 days; considering the classification of the cases, $76.0 \%(n=237)$ were considered Unspecified Early Congenital Syphilis. Conclusion: With this data, we can conclude that congenital syphilis still persists as a public health problem, associated with greater social vulnerability and failures in prenatal care.

Keywords: Congenital Syphilis, HIV infections, Infant Newborn.

\section{RESUMEN}

Objetivo: Investigar la prevalencia de recién nacidos con infección de sífilis y la exposición al VIH atendidos en un hospital público en Belém do Pará. Métodos: Estudio transversal cuantitativo, descriptivo y retrospectivo que utiliza datos secundarios de los registros de pacientes. El número total de neonatos ingresados en el hospital en lo período en cuestión fue de 8.075 pacientes. Resultados: Se incluyeron en este estudio 312 neonatos con diagnóstico de Sífilis congénita en el período de investigación; del total de incluidos, $7,1 \%(n=22)$ fueron expuestos al $\mathrm{VIH}$, caracterizando la coinfección. En cuanto al género, el 53,8\%

\footnotetext{
${ }^{1}$ Escola de Enfermagem Magalhães Barata da Universidade do Estado do Pará (UEPA). Belém - Pará.

${ }^{*}$ E-mail: marcosrisuenho.1@hotmail.com
}

SUBMETIDO EM: 5/2019

ACEITO EM: 6/2019

PUBLICADO EM: 7/2019

REAS | Vol. Sup.29 | e1028 | DOI: https://doi.org/10.25248/reas.e1028.2019 Página 1 de 9 
( $n=167)$ eran hombres y el $75,0 \%(n=234)$ estaban en el rango de edad de 0 a 10 días; considerando la clasificación de los casos, el 76,0\%(n=237) fue considerada Sífilis Congénita Temprana no Especificada. Conclusión: Con estos datos, se concluye que la sífilis congénita todavía persiste como problema de salud pública, asociada a la mayor vulnerabilidad social y fallas en la asistencia prenatal.

Palabras clave: Sífilis Congénita, Infecciones por VIH, Recién Nacido.

\section{INTRODUÇÃO}

As infecções sexualmente transmissíveis (ISTs) na gravidez, são consideradas um grave problema da Saúde Pública comuns em todo o mundo. Dessa forma, foram eleitas para trabalhar neste estudo a Sífilis Congênita e o Vírus da Imunodeficiência Humana (HIV) por apresentarem uma alta prevalência em adultos na idade fértil, tanto em países desenvolvidos como nos subdesenvolvidos (SANZ SM e GUINSBURG R, 2008; NEWMAN L, et al., 2013).

Com base nos dados do Ministério da Saúde, observa-se que o número de casos de sífilis em gestantes e de sífilis congênita registrados no Brasil indica que o agravo ainda persiste, evidenciando que a prevenção da Sífilis Congênita ainda é um desafio aos serviços de saúde. Em 2011, a taxa de detecção da sífilis em gestantes foi calculada em 5 casos por 1000 nascidos vivos; com relação à sífilis congênita, a taxa de incidência, em 2011, foi de 3,3 casos por 1000 nascidos vivos (BRASIL, 2012a).

A Sífilis Congênita incide frequentemente em áreas com alta prevalência do HIV. Tanto a sífilis como a infecção pelo HIV ainda são consideradas agravos de grande repercussão em saúde pública de extensão mundial, sobretudo, nos países em desenvolvimento. São infecções que podem ser transmitidas pelo sexo desprotegido, por transfusão de sangue, compartilhamento de agulhas e de modo vertical. No entanto, na gravidez, essas infecções têm sido associadas a um número de desfechos adversos (GÓMEZ GB, et al., 2013; ENDRIS M, et al., 2015).

A Organização Mundial da Saúde (OMS) estima que mais de 1,4 milhões de mulheres grávidas estão sendo acometidas pela sífilis e HIV no mundo a cada ano. No contexto gestacional, a sífilis e o HIV não tratada e HIV na gravidez têm sido relatadas como responsáveis por causar efeitos adversos e desfechos de gravidezes em cerca de $50 \%$ e $20 \%$ dos casos respectivamente. Portanto, a sífilis é responsável por grande morbidade durante a gestação, resultando em desfechos negativos em mais de 50\% dos casos, tais como: perdas fetais (aborto espontâneo, óbito fetal), óbito neonatal precoce e complicações tardias nos nascidos vivos, sobretudo entre as gestantes não tratadas (HAWKES SJ, et al., 2013; BRASIL, 2015a).

O objetivo deste estudo foi conhecer a prevalência de Sífilis congênita e exposição ao HIV em neonatos atendidos no período de 2014 a 2016 em um Hospital Materno Infantil no Município de Belém, no Estado do Pará.

\section{METODOS}

Estudo transversal com abordagem quantitativa, descritiva e retrospectiva, com o objetivo de conhecer a prevalência da infecção pela sífilis e exposição pelo HIV em neonatos no hospital em estudo.

O estudo foi desenvolvido na Fundação Santa Casa de Misericórdia do Pará, centro de referência de assistência materno-infantil do Estado, cadastrado como hospital de referência do SUS no atendimento à gestante de alto risco e ao recém-nascido. Atualmente presta assistência aos 144 municípios do Estado do Pará, pertencente ao atendimento exclusivo ao Sistema Único de Saúde (SUS).

A amostra foi composta de 312 prontuários dos neonatos atendidos na unidade de terapia intensiva neonatal do hospital, nos anos de 2014 a 2016. Teve como critério de inclusão os prontuários de neonatos atendidos no hospital, de ambos os sexos, os quais apresentassem diagnóstico confirmado de infecção com sífilis e exposição ao HIV. 
Foram aplicados para coleta de dados a ficha de notificação e investigação de Sífilis Congênita e a ficha de notificação e investigação da criança exposta ao HIV, ambas do Sistema de Informação de Agravos de Notificação (SINAN).

Os dados foram tabulados em uma planilha do programa Microsoft Excel@ versão 2010, analisados individual e conjuntamente por meio de estatística descritiva, realizadas a partir das variáveis definidas para os neonatos como sendo: sexo, idade, diagnóstico clínico do paciente para sífilis e exposição ao HIV, tratamento específico para sífilis, vivo, realização do exame VDRL (Veneral disease research laboratory). As variáveis da mãe: idade, residência, ocupação, escolaridade, realizou pré-natal, diagnóstico clínico da mãe e teste HIV.

O projeto foi aprovado em 20/01/2017 pelo Comitê de Ética em Pesquisa com Seres Humanos do Curso de Graduação em Enfermagem "Escola Magalhães Barata" e firmado pelo Comitê de Ética em Pesquisa com Seres Humanos da FSCMPA com número CAAE: 62053716.3.0000.5170 com número de parecer no1.895.342.

\section{RESULTADOS}

Foram internados na FSCMPA nos anos de 2014, 2015 e 2016 um total de 8075 pacientes neonatos com média de 2691 ao ano. No estudo foram incluídos 95,7\% ( $n=312)$ neonatos com diagnóstico de Sífilis congênita no período do estudo, deste total de neonatos com sífilis, 7,1\% ( $n=22)$ foram expostos ao HIV.

A faixa etária das mães dos neonatos ficou com maior ocorrência entre 21 a 30 anos correspondendo a 47,1\% ( $n=147)$ seguido de 14 a 20 anos com 34,6\% ( $n=108)$ e 13,5\% $(n=42)$ mães na faixa dos 31 a 42 anos.

Tratando-se da cor, foi encontrado nos registros das mães dos neonatos uma prevalência de mulheres consideradas pardas com $9,6 \%(n=30)$ e as consideradas brancas $0,4 \%(n=2)$ chamando a atenção o número de prontuários sem informação registrada com $89,4 \%$ ( $n=279)$ dos casos inviabilizando uma análise.

No Gráfico 1, são distribuídos os casos de mães de neonatos por procedência dos internados no período do estudo.

Gráfico 1 - Distribuição de mães de neonatos por procedência no Estado do Pará, no período de 2014 a 2016 na FSCMPA.

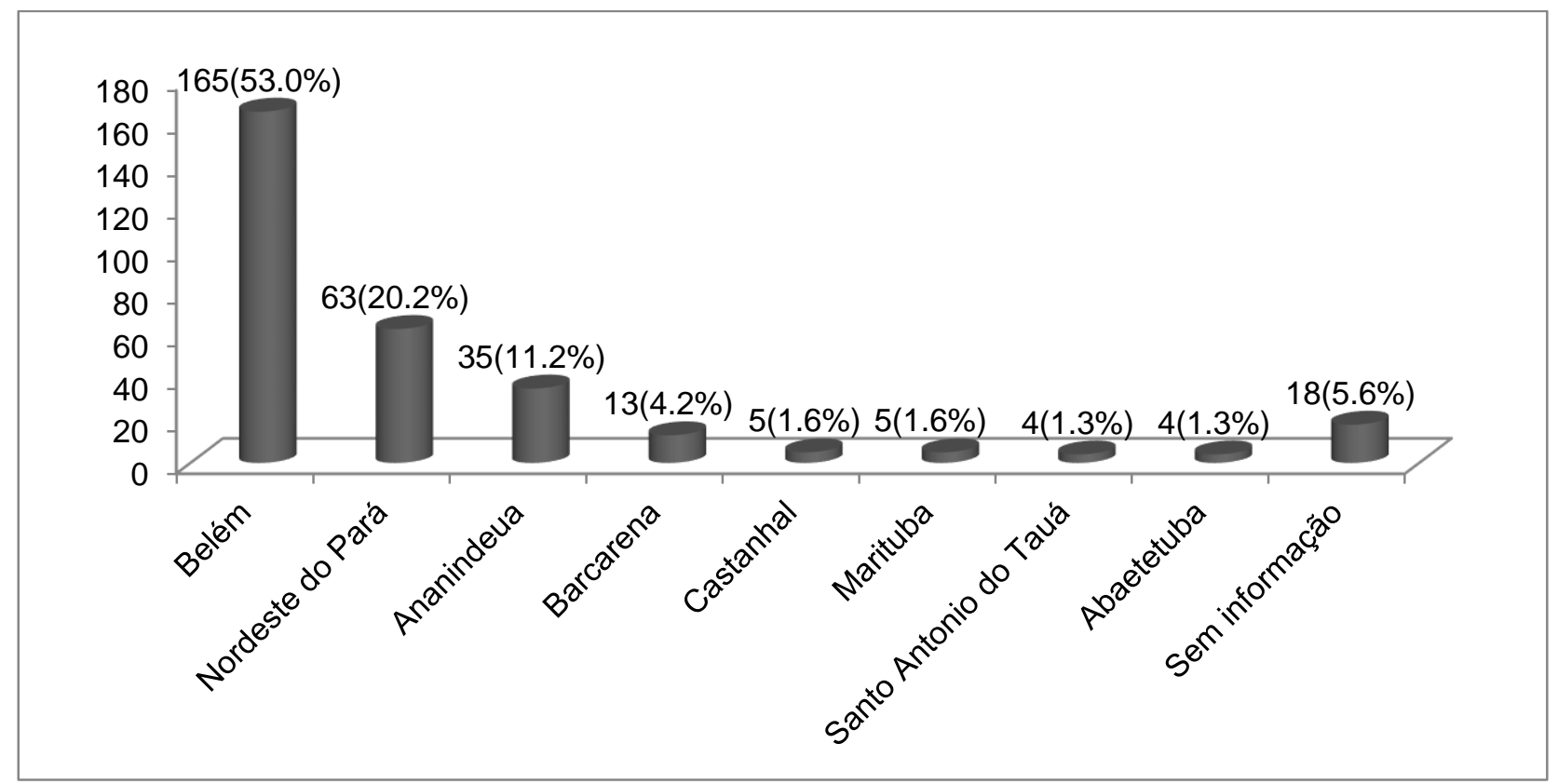

Fonte: Serviço de Arquivo Médico e Estatístico da FSCMPA. 
Com relação a vida profissional das mães dos neonatos foi verificado que das 312 analisadas, $44,2 \%$ $(n=138)$ eram Do Lar, considerado a maior prevalência observada. Chama a atenção na consulta dos prontuários a observação de dados sem informação, neste caso foi de $44.2 \% \quad(n=138)$ dos prontuários consultados. Quanto a realização do pré-natal, os resultados mostram que a maioria $79,2 \% \quad(n=247)$ realizaram pré-natal e 15,1\% ( $n=47)$ não realizaram. Destaca-se nos dados as mães com coinfecção - Sífilis e HIV, um total de $7,1 \%(n=22)$ mães soropositivas ao vírus HIV, concordando que 22 neonatos foram expostos ao HIV.

Dos prontuários analisados no período de 2014 a 2016 observou-se um total de prontuários sem fichas SINAN de $79,5 \%(n=248)$ e $20,5 \%(n=64)$ com fichas no prontuário. Predominou o sexo masculino com $53,8 \%(n=168)$ e o sexo feminino $45,2 \%(n=141)$. Essa distribuição mostra que de acordo com a faixa etária, houve maior prevalência de $75 \%(n=234)$ dos casos na faixa etária do neonato de $0-10$ dias, e $21,2 \%$ ( $n=66)$ entre 11 a 20 dias apenas $1,0 \%(n=3)$ maior que 20 dias.

Ao tratar-se da classificação como é dada nos casos de Sífilis congênita na FSCMPA, obteve-se uma maior ocorrência de sífilis congênita precoce não especificada com $76,0 \% \quad(n=237)$ toda classificação é mostrada no Gráfico 2.

Gráfico 2 - Distribuição dos tipos de classificação dos casos de Sífilis dos neonatos internados na FSCMPA no período de 2014 a 2016.

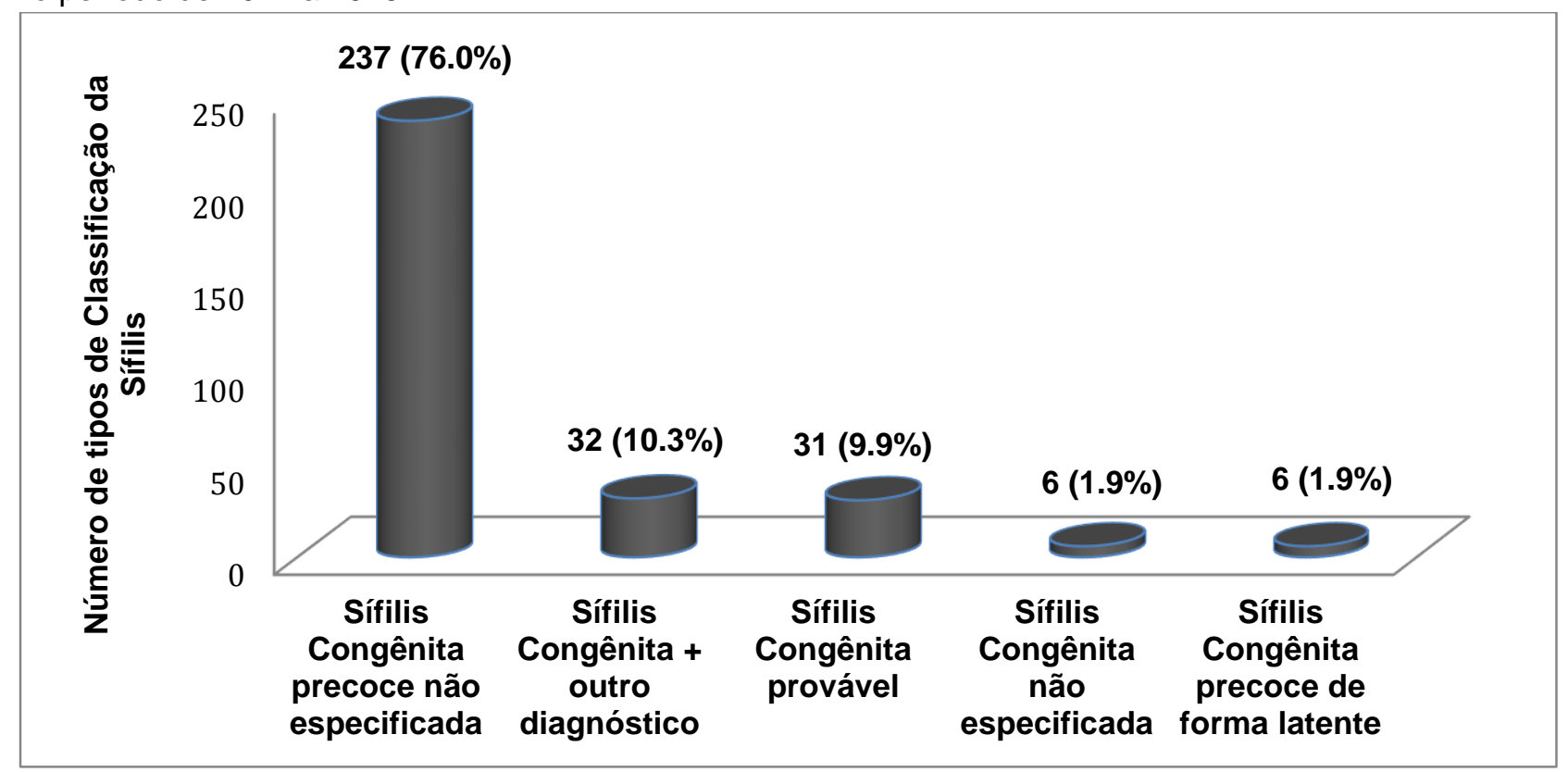

Fonte: Serviço de Arquivo Médico e Estatístico da FSCMPA.

Importante ressaltar que o diagnóstico de sorologia pelo VDRL foi realizado em 289 neonatos, destes, foi reagente em 50,3\% ( $n=157)$ e a titulação mínima considerada foi de $1: 2$ com $23,1 \%(n=72)$ como mostra as Tabelas 1 e 2.

Tabela 1 - Distribuição dos casos de Sífilis com diagnóstico do VDRL dos neonatos internados na FSCMPA no período de 2014 a 2016.

\begin{tabular}{l|c|c}
\hline Diagnóstico do VDRL & № & $\%$ \\
\hline Não Reagente & 132 & 42.3 \\
\hline Reagente & 157 & 50.3 \\
\hline Sem Informação & 23 & 7.4 \\
\hline Total & 312 & 100.0 \\
\hline
\end{tabular}

Fonte: Serviço de Arquivo Médico e Estatístico da FSCMPA. 
Tabela 2 - Distribuição dos casos de Sífilis com diagnóstico do VDRL e suas titulações dos neonatos internados na FSCMPA no período de 2014 a 2016.

\begin{tabular}{c|c|c}
\hline Resultado do VDRL em Título & № & $\%$ \\
\hline $01: 02$ & 72 & 23.1 \\
\hline $01: 04$ & 63 & 20.2 \\
\hline $01: 08$ & 38 & 12.2 \\
\hline $01: 16$ & 24 & 7.7 \\
\hline $01: 32$ & 18 & 5.7 \\
\hline$>1: 64$ & 11 & 3.5 \\
\hline Sem Informação & 86 & 27.6 \\
\hline Total & 312 & 100.0 \\
\hline
\end{tabular}

Fonte: Serviço de Arquivo Médico e Estatístico da FSCMPA.

No tratamento dos neonatos foi evidenciado uma diferença importante, predominando o tratamento com Penicilina G Cristalina com 45,8\% ( $n=143)$, com Cefalosporina de quarta geração $24,5 \%(n=76)$ e com Cefalosporina de $3^{\underline{a}}$ geração $19,6 \%$ ( $\left.n=61\right)$ além da associação das drogas nos outros casos, Tabela 3.

Tabela 3 - Distribuição do tratamento dos casos de Sífilis dos neonatos internados na FSCMPA no período de 2014 a 2016.

\begin{tabular}{|c|c|c|}
\hline TRATAMENTO & № & $\%$ \\
\hline Penicilina G Cristalina & 143 & 45.8 \\
\hline Cloridrato de Cefepima & 76 & 24.5 \\
\hline Ceftriaxona & 61 & 19.6 \\
\hline Penicilina G Benzatina+ Cloridrato de Cefepima & 5 & 1.6 \\
\hline Cloridrato de Cefepima +Zidovudina+Nevepirina & 3 & 1.0 \\
\hline Penicilina G Benzatina+Ceftriaxona & 3 & 1.0 \\
\hline Penicilina G Cristalina + Zidovudina + Neveripina & 3 & 1.0 \\
\hline Ceftriaxona Sódica + Penicilina G Benzatina & 2 & 0.6 \\
\hline Cloridrato de Cefepima + Gentamicina & 2 & 0.6 \\
\hline Cloridrato de Cefepima+ Gentamicina + Metronidazol & 1 & 0.3 \\
\hline Ceftriaxona Sódica +Zidovudina+ Nevepiropina & 1 & 0.3 \\
\hline Ceftriaxona Sódica+ Zidovudina + Penicilina G Benzatina & 1 & 0.3 \\
\hline Ceftriaxona Sódica + Cloridrato de Cefepima & 1 & 0.3 \\
\hline Ceftriaxona Sódica + Penicilina G Benzatina + Tobramicina & 1 & 0.3 \\
\hline Nevirapina & 1 & 0.3 \\
\hline Oxacilina + Gentamicina + Cloridrato de Cefepima & 1 & 0.3 \\
\hline Penicilina G Cristalina + Metronidazol + Gentamicina & 1 & 0.3 \\
\hline Penicilina G Benzatina & 1 & 0.3 \\
\hline Penicilina G Cristalina + Zidovudina + Ampicilina & 1 & 0.3 \\
\hline Penicilina G Cristalina + Gentamicina & 1 & 0.3 \\
\hline Sem informação & 3 & 1.0 \\
\hline Total & 312 & 100.0 \\
\hline
\end{tabular}

Fonte: Serviço de Arquivo Médico e Estatístico da FSCMPA. 


\section{DISCUSSÃO}

Considera-se que as infecções de origem congênita possuem diferentes fatores relacionados, como: características da saúde materna, peculiaridades microbiológicas e patogênicas inerentes a determinado patógeno, integridade da placenta e um relevante fator, idade gestacional no momento da obtenção da infecção materna, bem como o caráter biológico. Relacionam-se também com o cenário socioeconômico político e cultural favorável ou não a prevenção de doenças, pois em países desenvolvidos houve a diminuição de doenças infectocontagiosas, uma vez que essas são preveníveis (LAFETÁ GRK, et al., 2016).

No hospital foram evidenciados 312 casos de sífilis congênita em recém-nascidos vivos e coinfecção em 22 neonatos (sífilis e expostos ao HIV). De acordo com o Boletim Epidemiológico da Sífilis de 2016, no ano de 2015 foi observada uma taxa de incidência de 6,5 casos para cada mil nascidos vivos, sendo que especificamente na região Norte a taxa foi de 4,4 casos a cada mil nascidos vivos.

Constatou-se que nos últimos 10 anos houve um aumento na taxa de casos de sífilis congênita no Brasil (de 2,2 casos/mil nascidos vivos para 6,5 casos/mil nascidos vivos). Os dados apresentados no presente estudo e no Boletim epidemiológico de 2016 apontam que ainda não se atingiu a meta estabelecida pelo MS com o Pacto pela Saúde entre a OMS, a qual era a de eliminar a sífilis congênita até o ano de 2015 (BRASIL, 2016c; LAFETÁ GRK, et al., 2016).

Neste estudo, a faixa etária predominante das mães foi entre 21 a 30 anos correspondendo a 47,1\%, seguido de 14 a 20 anos com, 34,6\%. Dados que demonstram a existência de ampla variação quanto a idade que vai desde a adolescência até 40 anos. Este resultado concorda com um estudo de coorte nacional realizado no ano de 2011 a 2012 com 23.894 mulheres, que objetivou analisar a cobertura a abrangência de testagem para sífilis durante o Pré-natal e a prevalência de sífilis na gestação, o qual apresentou idade dominante das genitoras de 20 a 34 anos.

Dados esses confirmados pelo Boletim Epidemiológico de Sífilis do ano de 2016, que obteve a prevalência da idade das nutrizes de 20 a 29 anos, com $25,7 \%$ dos casos e de 30 a 39 anos correspondentes a $52,5 \%$. Ou seja, a predominância é na idade reprodutiva a partir dos 20 anos de idade, o que foi encontrado em estudos recentes (DOMINGUES MSMR, et al., 2014; BRASIL, 2016).

Tratando-se da cor, que foi encontrada nos registros das mães dos neonatos obteve-se a prevalência de mulheres consideradas pardas com 9,6\% ( $n=30)$, impossibilitando uma análise mais precisa, já que o número de prontuários sem esta informação foi de $89,7 \%(n=280)$. Segundo dados do Boletim Epidemiológico de sífilis do ano de 2016 destacou-se a grande predominância de genitoras pardas, correspondendo a 47,8\% ( $n=7281$ ) dos casos, e em seguida as brancas com 30,4\% $(n=4613)$ dos casos de sífilis (BRASIL, 2016).

A procedência das mães dos neonatos predominou na região metropolitana de Belém (53\%) seguido dos municípios no Nordeste do Estado do Pará. Já os dados extraídos relacionados à vida profissional das mães com sífilis verificou-se que $44,2 \%$ dessas mulheres eram do lar, e concomitantemente $44,2 \%$ dos prontuários estavam sem essa informação. Essa prevalência de profissões do lar concorda com um estudo realizado no município de Campo Grande no período de outubro de 2008 a setembro de 2009, com gestantes com diagnóstico de sífilis confirmada, o qual obteve $62,7 \%$ das mães com ocupação do lar (HILDERBRAND CPLV, 2010).

No que se refere aos $44,2 \%$ de falta de informações sobre a profissão, assim como a ausência total de informações sobre a escolaridade materna, encontra-se um viés de estudo, tanto a falta de notificação, quanto o preenchimento incorreto das Fichas de Notificação do SINAN, o que é uma realidade e problemática nos serviços de saúde, o que dificulta os estudos de cunho epidemiológico de doenças de notificação compulsória (BRASIL, 2012a).

Com relação ao cuidado pré-natal, neste aspecto inclui a promoção da saúde, a prevenção e o tratamento dos problemas que possam ocorrer durante o período gestacional, após o parto. Dessa forma garantindo a qualidade da assistência prestada pelo serviço de saúde e pelos profissionais, como fator essencial para a adesão das mulheres ao pré-natal, incentivando-as a realizá-lo (BRASIL, 2012b). 
Destaca-se que o acesso ao serviço de pré-natal foi de 79,2\% (247), embora 15,1\% (47) não tenham realizado. Este estudo apresentou dados equivalentes aos dados nacionais. Nos dados nacionais cerca de $78,4 \%$ das mães de neonatos com diagnóstico de sífilis congênita realizaram o pré-natal no ano de 2015 , enquanto que $15 \%$ não realizaram (BRASIL, 2016a).

Observa-se que os dados referentes a realização do pré-natal são altos. No entanto, ainda não segue de forma eficiente, tal como o recomendado pelo MS. É preconizado que a cobertura do pré-natal deve ser adequada em quantidade e qualidade e abranger a todas as gestantes.

A assistência do pré-natal deve garantir a captação precoce da gestante (até 120 dias da gestação), a realização de no mínimo sete consultas e a oferta de exames laboratoriais básicos como o VDRL, que deve ser investigado no mínimo duas vezes durante a gestação (BRASIL, 2012b).

Cunha CRA (2015), conclui que mesmo com as melhorias, o tratamento continua como o grande desafio para o controle da sífilis no país e constata que mais da metade das parturientes diagnosticadas durante 0 pré-natal continuavam com a infecção quando admitidas para o parto, mesmo existindo medicação de eficácia comprovada e com disponibilidade na rede pública.

Das 312 mães analisadas no presente estudo, 7,1\% (22) possuíam o vírus HIV ou estavam expostas ao mesmo. Sanz SM e Guinsburg R (2008) realizaram um estudo no mesmo hospital da atual pesquisa, no período de agosto a setembro de 2000 com casuística de 1151 gestantes, no qual encontraram soropositivas simultaneamente para sífilis e o vírus HIV em $0,7 \%$ e destacaram o aumento gradativo de mulheres com HIV ao longo do tempo e assim a tendência do aumento de casos a nível estadual e nacional, o que é ratificado pelos dados encontrados no presente estudo.

Um outro estudo apresenta dados que se aproximam dos resultados da pesquisa em questão, realizado em Porto Alegre, por Acosta LMW, et al., (2016) com dados obtidos a partir das notificações de gestantes portadoras de HIV e crianças expostas no período de 2010 a 2013, teve como um universo amostral 1.500 gestantes, desse universo um quantitativo de 155 grávidas manifestou coinfecção, no qual a taxa de coinfecção geral de HIV e Sífilis foi de 10,2\%.

Estudos mostram que a sífilis é a coinfecção mais prevalente em gestantes com o HIV, e além dos eventos vinculados a morbidade de cada patologia, a presença da coinfecção facilita a transmissão vertical tanto do HIV quando da sífilis. Os pacientes que apresentam as duas infecções simultaneamente indicam alterações tanto na resposta imune humoral do paciente quanto na resposta ao tratamento para a sífilis (ACOSTA LMW, et al., 2016;

Dentre os resultados encontrados $6,8 \%(n=21)$ das mães são usuárias de drogas, dessas, $6 \%(n=1)$ têm a infeção de Sífilis e HIV. Há comprovações de que a assistência pré-natal diminui de maneira eficaz os resultados negativos da utilização de drogas ilícitas durante o período gestacional. O que evidencia a necessidade de traçar o perfil socioeconômico desta população acometida (COUTINHO T, et al., 2014).

Relacionado a questão de notificação SINAN, no presente estudo do período de 2014 a 2016, 248 casos não foram notificados, ou seja, $79,5 \%$ de subnotificação. Fato este bastante encontrado em pesquisas, quando trata-se de estudos secundários. Como exemplo tem-se um estudo realizado em Belo Horizonte que estimou a subnotificação de Sífilis Congênita em 55, 2\% (NONATO MS, et al., 2015)

A notificação influencia diretamente no controle da Sífilis Congênita e a subnotificação pode ser levada em consideração como uma indicação indireta de baixa qualidade de atendimento do pré-natal, pois a notificação de sífilis na parturiente permitiria o controle da transmissão vertical da Sífilis Congênita, investigar 0 comportamento da patologia entre as nutrizes o que permitirá o planejamento de serviços de saúde, e posteriormente implantações e implementações de intervenções de saúde (NONATO MS, et al., 2015).

Além da subnotificação das fichas SINAN de sífilis congênita, não foi encontrado nenhuma ficha de notificação da criança exposta ao HIV, assim como nenhum registro sobre a realização de exames pertinentes a investigação da contaminação do neonato pelo vírus da imunodeficiência humana. 
A faixa etária predominante do diagnóstico de sífilis congênita neste estudo foi de 0 a 10 dias correspondente a $75 \%$ recém-nascidos, de 11 a 22 dias correspondente a $21,2 \%$.

Nos dados do Boletim Epidemiológico da Sífilis de 2016 identificou de 1988 a junho de 2016 com 142.961 casos notificados de sífilis congênita em menores de um ano de idade, destes 5,8\% (8.363) são da região Norte (BRASIL, 2016b).

Quanto a realização do exame VDRL nos neonatos, encontrou-se o registro de 289 recém-nascidos, dentre estes $42,3 \%(n=132)$ foram não reagentes, 50,3\% $(n=157)$ reagentes e 7,4\% $(n=23)$ sem registro algum da realização do teste não treponêmico. Com títulos entre 1:4 a 1:64.

Coutinho RLC (2014), com sua pesquisa no Ceará, identificou que 51,96\% ( $n=409)$ dos recém-nascidos de mães com sífilis congênita, nascidos no período de 2007 a 2012, apresentaram VDRL reagente em baixas titulações e 3,16\% não realizaram o VDRL, fato este consonante ao presente estudo.

O Ministério da Saúde preconiza que todos os testes devem ser realizados com o sangue do neonato, contraindicando a utilização de amostra de sangue obtido do cordão umbilical, pois esse tipo de amostra contém uma união do sangue da criança e da mãe e, portanto, anticorpos de ambos.

Devido à falta de registro, no prontuário, quanto a origem do material coletado para fins de diagnóstico percebe-se a fragilidade das informações coletadas sobre as titulações dos neonatos, pois uma vez que a coleta tenha sido realizada de forma incorreta pode haver a sugestão de um falso-negativo ou falso-positivo (BRASIL, 2016b).

Outro dado interessante foi a questão do tratamento, neste estudo o notou-se a maior administração de Penicilina G Cristalina, tratamento de primeira escolha preconizado pelo ministério da saúde, e o uso de Cefalosporinas de $3^{\underline{a}}$ e $4^{\underline{a}}$ gerações, $19,6 \%$ e $24,5 \%$ concomitantemente. O estudo foi realizado do mês de janeiro de 2014 até o mês de dezembro de 2016.

Sugere-se que a utilização dos medicamentos que não são de primeira escolha, ocorreu em função de um desabastecimento da droga a nível nacional, uma vez que foi encontrada a observação em um prontuário que justificava o uso de Ceftriaxona Sódica pela indisponibilidade da medicação no hospital (BRASIL, 2016c).

No estudo foi encontrada uma porcentagem de $7,1 \%$ de recém-nascidos infectados com sífilis e expostos ao HIV, os quais receberam o tratamento padrão para exposição ao HIV segundo o protocolo clínico e diretrizes terapêuticas para o manejo de infecção pelo HIV em crianças e adolescentes.

Em tal protocolo, é indicado o uso por solução oral, desde o nascimento, a Zidovudina e Nevirapina por mais de três semanas, além da não amamentação (BRASIL, 2014).

Nesse sentido, a ocorrência de sífilis congênita e exposição ao HIV em neonatos demonstram que, no contexto analisado, ambas se relacionam a fatores socioeconômicos, culturais, uso de drogas e, sobretudo, a não realização do pré-natal.

Portanto, é fundamental investir na implementação de políticas públicas voltadas a assistencial pré-natal, de modo a promover a melhoria da oferta de exames sorológicos e tratamento oportuno das gestantes (SANZ SM e GUINSBURG R, 2008).

\section{CONSIDERAÇÕES FINAIS}

Com a realização deste estudo, foi possível investigar a prevalência de neonatos com infecção por sífilis e exposição ao HIV atendidos em um hospital público de Belém do Pará. A prevalência dessas infecções está relacionada com diferentes fatores, como: características da saúde materna, peculiaridades microbiológicas e patogênicas inerentes a determinado patógeno, integridade da placenta e um relevante fator, idade gestacional no momento da obtenção da infecção materna; mas, também tem relações com fatores socioeconômicos e culturais. Nesse contexto, é de suma importância o papel da atenção primária, durante o pré-natal, oferecer ações assistenciais que visem a detecção da Sífilis e do HIV na gestante, de modo a prevenir precocemente a transmissão vertical dessas doenças. 


\section{REFERÊNCIAS}

1. ACOSTA LMW, et al. Coinfecção HIV/sífilis na gestação e transmissão vertical do HIV: um estudo a partir de dados da vigilância epidemiológica. Revista Panamericana de Salud Publica, 2016; 40(6): 435-42.

2. BRASIL. Ministério da Saúde. Secretaria de Vigilância em Saúde. Transmissão Vertical do HIV e Sífilis: Estratégias para redução e eliminação. Brasília: Ministério da Saúde; 2012a; 20p.

3. BRASIL. Ministério da Saúde. Secretaria de Atenção à Saúde. Departamento de Atenção Básica. Atenção ao prénatal de baixo risco. Brasília: Ministério da Saúde; 2012b; 318p.

4. BRASIL. Ministério da Saúde. Secretaria de Vigilância em Saúde. Departamento de DST, AIDS e Hepatites Virais. Protocolo Clínico e Diretrizes Terapêuticas Para Manejo da Infecção Pelo HIV em Crianças e Adolescentes. Brasília: Ministério da Saúde; 2014; 240p.

5. BRASIL. Ministério da Saúde. Secretaria de Ciência, Tecnologia e Insumos Estratégicos Esplanada dos Ministérios. Ceftriaxona para tratamento de sífilis em gestantes com alergia confirmada a penicilina. Ano I. n. 153. Brasília: Ministério da Saúde, 2015a; 16p.

6. BRASIL. Ministério da Saúde. Secretaria de Vigilância em Saúde. Departamento de DST, AIDS e Hepatites Virais. Boletim Epidemiológico - Sífilis. Ano I. n. 35. Brasília: Ministério da Saúde; 2016a; 28p.

7. BRASIL. Ministério da Saúde. Secretaria de Vigilância em Saúde. Departamento de Vigilância, Prevenção e Controle das Doenças Sexualmente Transmissíveis, Aids e Hepatites Virais. Manual Técnico para Diagnóstico da Sífilis. Brasília: Ministério da Saúde, 2016b; 52 p.

8. BRASIL. Ministério da Saúde. Nota Informativa Conjunta, 19 de agosto de 2016. Orienta sobre o tratamento de sífilis congênita e neurossífilis em recém-nascidos somente na disponibilidade de penicilina G cristalina ou potássica. Brasília: Ministério da Saúde; 2016c; 7p.

9. COUTINHO RLC. Sífilis Congênita: panorama do agravo em um hospital de ensino. Dissertação (Mestrado) Universidade Federal do Ceará, Fortaleza, 2014; 85p.

10. COUTINHO T, et al. Assistência pré-natal às usuárias de drogas ilícitas. FEMINA, 2014; 42(1): 11-18.

11. CUNHA CRA. Sífilis em Parturientes do Brasil: prevalência e fatores associados. Dissertação (mestrado) Universidade de Brasília, Distrito Federal, 2015; 82p.

12. DOMINGUES MSMR, et al. Prevalência de Sífilis na Gestação e Testagem Pré-Natal: Estudo Nascer no Brasil. Revista de Saúde Pública, 2014; 48(5):766-774.

13. ENDRIS M, et al. Seroprevalence of syphilis and human immunodeficiency virus infections among pregnant women who attend the University of Gondar Teaching Hospital, Northwest Ethiopia: a cross sectional study. BMC Infectious Diseases, 2015; 15: 111.

14. GÓMEZ GB, et al. Untreated maternal syphilis and adverse outcomes of pregnancy: a systematic review and metaanalysis. Bulletin of the World Health Organization, 2013; 91:217-26.

15. HAWKES SJ, et al. Early Antenatal Care: Does It Make a Difference to Outcomes of Pregnancy Associated with Syphilis? A Systematic Review and Meta-Analysis. PLOS ONE, 2013; 8(2): 1-7.

16. HILDERBRAND CPLV. Sífilis Congênita: fatores associados ao tratamento de gestantes e seus parceiros. Dissertação (mestrado) - Fundação Oswaldo Cruz, Escola Nacional de Saúde Pública Sergio Arouca, Rio de Janeiro, 2010; 85p.

17. LAFETÁ GRK, et al. Sífilis Materna e Congênita, Subnotificação e Difícil controle. Revista Brasileira de Epidemiologia, 2016; 19(1): 63-74.

18. NEWMAN L, et al. Global estimates of syphilis in pregnancy and associated adverse outcomes: analysis of multinational antenatal surveillance data. PLOS Medicine, 2013; 10(2): 1-10.

19. NONATO MS, et al. Sífilis Na Gestação e Fatores Associados Sífilis Congênita em Belo Horizonte-MG, 2010-2013. Revista Epidemiologia e Serv. Saúde, Brasília, 2015; 24(4): 681-694.

20. SANZ SM, GUINSBURG R. Prevalência da soropositividade para Sífilis e HIV em gestantes de um Hospital de Referência Materno Infantil do Estado do Pará. Revista Paraense de Medicina, 2008; 22(3): 1-11. 